\title{
Article \\ Terahertz Absorber with Graphene Enhanced Polymer Hemispheres Array
}

\author{
Alesia Paddubskaya ${ }^{1}{ }^{(}$, Nadzeya Valynets $^{1}$, Sergey Maksimenko ${ }^{1}{ }^{\circledR}$, Mukesh Kumar $^{2}{ }^{\circledR}$, Marian Baah ${ }^{2}$, \\ Markku Pekkarinen ${ }^{2}$, Yuri Svirko ${ }^{2}$, Gintaras Valušis ${ }^{3}$ (D) and Polina Kuzhir ${ }^{1,2, *}$ \\ 1 Laboratory of Nanoelectromagnetics, Institute for Nuclear Problems of Belarusian State University, \\ Bobruiskaya Str. 11, 220006 Minsk, Belarus; paddubskaya@gmail.com (A.P.); \\ Nadezhda.Volynets@gmail.com (N.V.); sergey.maksimenko@gmail.com (S.M.) \\ 2 Institute of Photonics, Department of Physics and Mathematics, University of Eastern Finland, \\ Yliopistokatu 7, 80101 Joensuu, Finland; mkkhatri32@gmail.com (M.K.); marian.baah@uef.fi (M.B.); \\ markku.pekkarinen@uef.fi (M.P.); yuri.svirko@uef.fi (Y.S.) \\ 3 Center for Physical Sciences and Technology, Sauletekio Av. 3, 10257 Vilnius, Lithuania; \\ gintaras.valusis@ftmc.lt \\ * Correspondence: polina.kuzhir@uef.fi; Tel.: +358-50-66-66-24
}

Citation: Paddubskaya, A.; Valynets, N.; Maksimenko, S.; Kumar, M.; Baah, M.; Pekkarinen, M.; Svirko, Y.; Valušis, G.; Kuzhir, P. Terahertz Absorber with Graphene Enhanced Polymer Hemispheres Array. Nanomaterials 2021, 11, 2494. https:// doi.org/10.3390/nano11102494

Academic Editor: Martijn Wubs

Received: 13 August 2021

Accepted: 21 September 2021

Published: 24 September 2021

Publisher's Note: MDPI stays neutral with regard to jurisdictional claims in published maps and institutional affiliations.

Copyright: (c) 2021 by the authors. Licensee MDPI, Basel, Switzerland. This article is an open access article distributed under the terms and conditions of the Creative Commons Attribution (CC BY) license (https:// creativecommons.org/licenses/by/ $4.0 /)$.

\begin{abstract}
We propose an original technique for the fabrication of terahertz (THz) metasurfaces comprising a 3D printed regular array of polymer hemispheres covered with a thin conductive layer. We demonstrate that the deposition of a thin metal layer onto polymer hemispheres suppresses the $\mathrm{THz}$ reflectivity to almost zero, while the frequency range of such a suppression can be considerably broadened by enhancing the structure with graphene. Scaling up of the proposed technique makes it possible to tailor the electromagnetic responses of metasurfaces and allows for the fabrication of various components of $\mathrm{THz}$ photonics.
\end{abstract}

Keywords: graphene; metasurface; terahertz; absorption; electromagnetic wave

\section{Introduction}

Broadband and frequency-tunable electromagnetic (EM) absorbers are widely used for the fabrication of a variety of EM components including polarizers, filters, attenuators and other devices of the microwave and THz photonics [1-4]. Sculpturing of conductive surfaces is one of the most developed techniques to enhance absorption at the microwave and $\mathrm{THz}$ frequencies [5]. Conventional EM absorbers relies on metal surface covered with subwavelength pyramid-like structures organized in a square or hexagonal lattice [6]. In such a structure, enhancement of the microwave absorption is achieved via "smoothing" the metal-vacuum interface when the incident EM wave "feels" the gradual change of the impedance from vacuum to the material one. However, efficient absorption requires pyramids taller than the quarter of the wavelength [7]. That is, the height of the pyramidal structure on the surface should exceed $2 \mathrm{~m}$ to efficiently absorb radiation at the frequency of $30 \mathrm{MHz}$ or should have the height of $10 \mathrm{~cm}$ to absorb EM waves in the frequency range of $8-40 \mathrm{GHz}$. The fabrication of such structures for the mega- and gigahertz range is well documented and their cost rapidly decreases due to development of the 3D printing technology [8]. However, in the THz range, the height of the pyramidal structure should be in the sub-millimeter range, i.e., a costly and time-consuming lithography techniques are needed to control the shape of the surface corrugations [9].

The above problems can be partially solved by replacing sculptured metal structure with graphene or other ultrathin carbon films possessing high absorption ability [10] in the THz range. High conductivity [11], record carrier mobility of graphene [12] combined with optical transparency at room temperature [13] and the pronounced sensitivity of its electronic properties to environment and external forces [14] allow one to use graphene- 
based absorbers for detecting tiny variations of the EM field, e.g., for using them in bolometers and $\mathrm{THz}$ sensors.

Placing not-structured graphene sheet onto flat dielectric substrate supported by metallic back reflectors $[15,16]$ is a well-established approach to achieve perfect absorption of EM radiation at particular frequency. There have also been several techniques developed for incorporating graphene into semiconductor and metal 3D patterns [17-20] and for using multilayer graphene islands or nanoribbons [21,22], which can be synthesized on the dielectric surfaces via a sacrificial metal layer.

However, the intrinsic planar structure of graphene imposes severe limitations on the using it for sensing [23,24]. This is due to the fact that the EM response of graphene-based sensing devices shows pronounced frequency dependence, which is governed solely by the graphene insulating support. Broadband EM absorbers require either patterning of graphene, i.e., making graphene-based metamaterial or using a multilayers approach. The fabrication of graphene-based metamaterial [25] involves time-consuming electron beam lithography and etching that make scaling up this approach a rather difficult task. Moreover, the EM response of such a metamaterial will be dependent not only on the graphene quality and doping level, but also on the quality of the graphene patterning process. The multilayer approach allows decreasing the thickness of the absorber by using a quarter wavelength thick Salisbury screen [26], which, however, suppresses the level of absorption substantially.

In this paper, we show that these difficulties can be overcome by enhancing the 3D metasurface with the flat graphene sheet. We propose a simple and scalable technique for fabrication of such graphene enhanced metasurfaces combining 3D printing, metal deposition through thermal evaporation, or magnetron sputtering and conventional graphene transfer processes.

We choose the periodic array of polymer hemispheres as a metasurface scaffold since it provides facile matching of the medium/air impedances to approach suppressing the reflection of EM radiation. The multiple reflections in between individual hemispheres couple the incident wave to the surface ones making the metasurface highly absorptive at certain frequencies, which depend on the diameter of hemisphere and periodicity of the array. Covering of the polymer corrugated surface with thin layer of metal (imaginary part of permittivity $\operatorname{Im} \varepsilon \gg 1$ ), which thickness $l$ is much smaller that the skin depth and the wavelength $l \sqrt{ } \varepsilon \ll \lambda$, should lead to substructional absorption of THz radiation [27].

We demonstrate that graphene enhanced 3D printed metasurfaces are capable to achieve almost zero reflectance and $>70 \%$ absorptance at the spectral range spanning from $200 \mathrm{GHz}$ to $1 \mathrm{THz}$ and can exhibit nearly perfect absorption at the frequencies of $0.8-1 \mathrm{THz}$.

\section{Electromagnetic Modelling}

In the course of the optimizing geometry of the structure in terms of the hemisphere diameter and the array periodicity, we performed numerical simulation of the EM response of the silica/polymer hemispheres/Ni and silica/polymer hemispheres/Ni/graphene metasurfaces in the CST Studio environment.

Figure 1 shows the transmittance $(T)$, reflectance $(R)$, and absorptance $(A)$ calculated for the square lattice array of polymer hemispheres covered with thin Ni layer and deposed on silica substrate. $\mathrm{SiO}_{2}$ is widely used in $\mathrm{THz}$ range as optical window being non-absorptive unlike conventional $(1-10 \mathrm{Om} / \mathrm{cm})$ silicon. Comparing the simulation results obtained for metasurfaces having periods of $400 \mu \mathrm{m}(\mathrm{a}-\mathrm{c})$ and $600 \mu \mathrm{m}(\mathrm{d}-\mathrm{f})$, one can see that the first one demonstrates a narrow absorptance band when the hemisphere diameter is bigger than $300 \mu \mathrm{m}$. In contrast, the metasurface composed of polymer hemispheres with a period of 600 microns is highly absorptive in the wide frequency range $250-600 \mathrm{GHz}$, when the hemispheres diameter is in the range from 350 to 550 microns. 


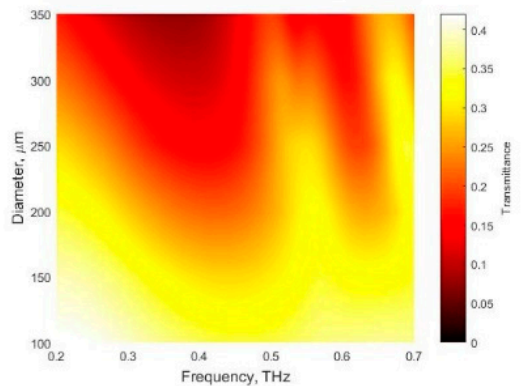

(a)

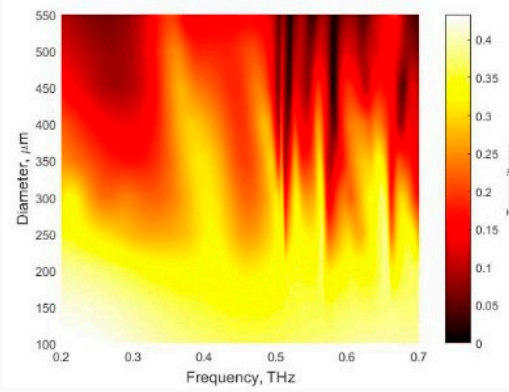

(d)

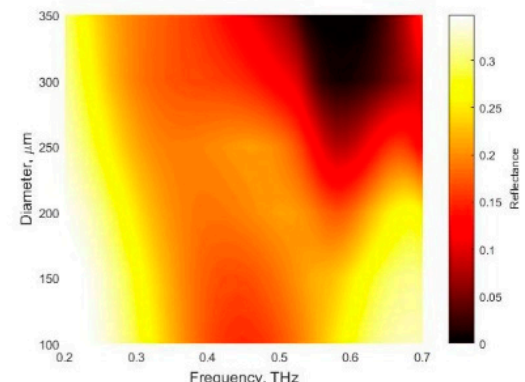

(b)

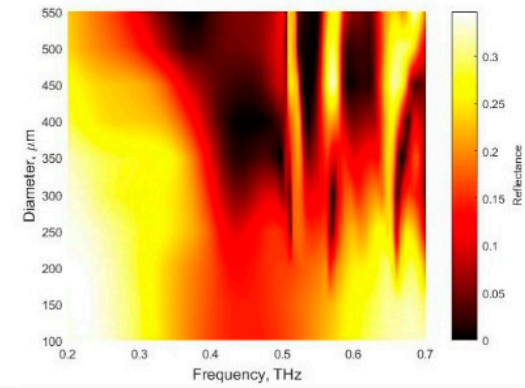

(e)

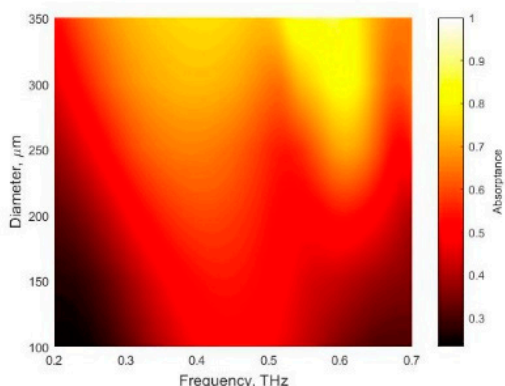

(c)

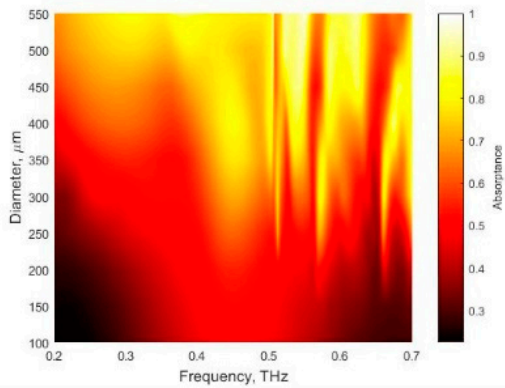

(f)

Figure 1. Transmittance $(T)$, reflectance $(R)$, and absorptance $(A=1-T-R)$ of multilayer system contained the array of polymer hemispheres covered with Ni layer (30 nm thick) and deposed on $\mathrm{SiO}_{2}$ substrate versus hemispheres diameter and frequency. The calculations were performed for the structure organized in the square lattice with $400 \mu \mathrm{m}(\mathrm{a}-\mathbf{c})$ and $600 \mu \mathrm{m}$ (d-f) period, respectively. The following parameters were used in the simulation: the polymer dielectric permittivity was 2.3; the dielectric permittivity of $\mathrm{SiO}_{2}$ substrate was set 2.7.

One can see from the spectra presented in Figure 2a that the EM response of the metasurface with the period 600 microns, comprising polymer hemispheres of 440 microns diameter covered with thin metal film, demonstrates a rather good absorption performance showing the average absorptance at the level of $75 \%$ and maximum absorptance of as high as $90 \%$ at resonance frequencies $0.38,0.54$ and $0.6 \mathrm{THz}$. It is worth noting that due to pronounced frequency dependence of $\mathrm{Ni}$ surface conductance in this frequency range, the $\mathrm{SiO}_{2} \backslash$ polymer hemispheres $\backslash \mathrm{Ni}$ structure is almost $40 \%$ transparent in the low-frequency edge of the THz spectra.

On the contrary to the thin Ni metal film, the surface conductivity of graphene is frequency independent in the $\mathrm{THz}$ domain [28] (i.e., one may expect that enhancing the metasurface with graphene may results in higher absorptance). Figure $2 b$ shows that placing graphene sheet onto the metasurface enhances absorptance to about $90 \%$ in the broad spectral range and $>95 \%$ at resonant frequencies of $0.52 \mathrm{THz}$ and $0.6 \mathrm{THz}$. The distribution of the electric field amplitude at a frequency of $0.6 \mathrm{THz}$ is presented in Figure $2 c, d$, and most clearly illustrates the enhancement effect. One may see that placing the graphene onto the metasurface leads to higher electric field amplitude at the surface of hemispheres and in the gap between the hemispheres indicating the higher absorption ability of graphene-enhanced architecture. 


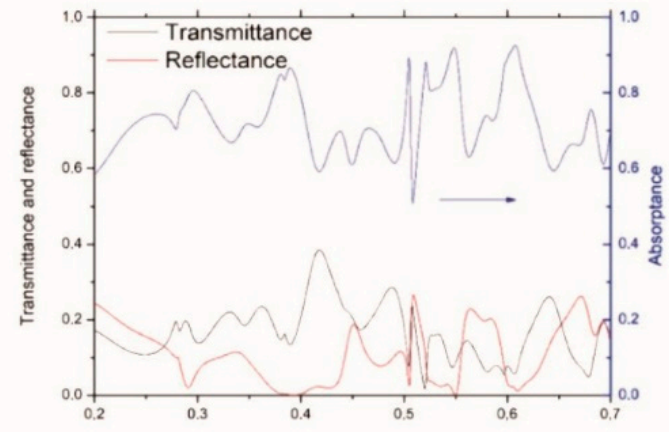

(a)

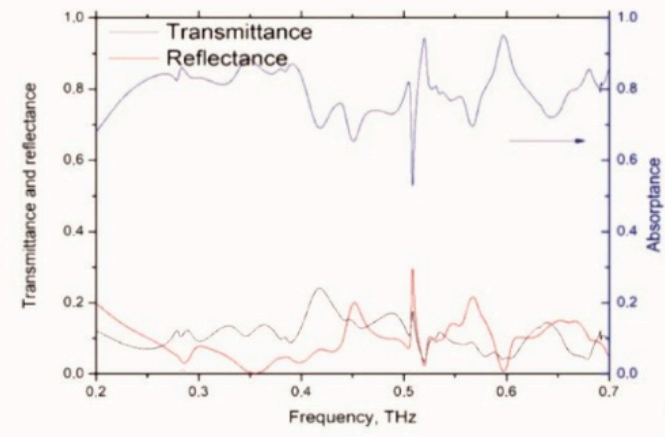

(b)

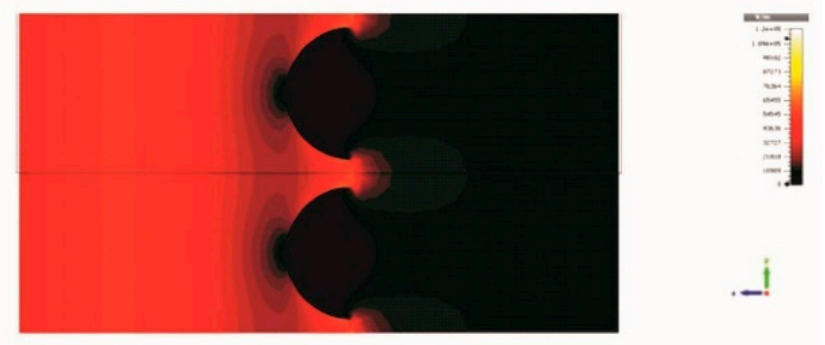

(c)

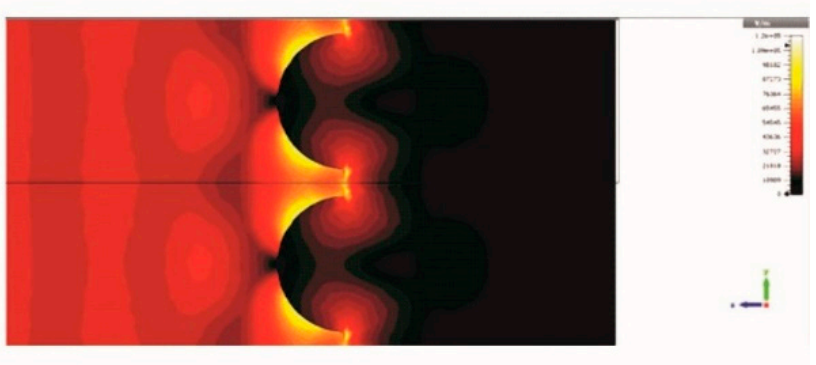

(d)

Figure 2. Frequency dependence of the transmittance $(T)$, reflectance $(R)$ and absorptance $(A)$ for $(\mathbf{a}) \mathrm{SiO}_{2} \backslash$ polymer hemispheres $\backslash \mathrm{Ni}$ and (b) $\mathrm{SiO}_{2} \backslash$ polymer hemispheres $\backslash \mathrm{Ni} \backslash$ graphene. (c) Distribution of the electric field amplitude at a frequency of $0.6 \mathrm{THz}$ at the surface of the structure $\mathrm{SiO}_{2} \backslash$ polymer hemispheres $\backslash \mathrm{Ni}$; (d) the same for the $\mathrm{SiO}_{2} \backslash$ polymer hemispheres $\backslash \mathrm{Ni} \backslash$ graphene. The following parameters were used in the calculations: the diameter and period of the hemispheres on the $\mathrm{SiO}_{2}$ surface are $440 \mu \mathrm{m}$ and $600 \mu \mathrm{m}$, respectively, the sheet conductance of graphene is $1.1 \mathrm{mS}$, the nickel sheet conductance is $3 \mathrm{mS}$.

\section{Materials and Methods}

\subsection{Printing}

In order to fabricate graphene enhanced THz metasurface, we employ a high-resolution layer-by-layer 3D printing technique [29-31] with 3D printer having three independent heads. Each head sends out drops of the liquid polymer LUX-Opticlear (Luxexcel, Alpharetta, GA, USA). The lateral size of the drop on the substrate $<20$ microns. The drops ejected by three heads form a $<5 \mu \mathrm{m}$ thick layer having prescribed lateral structure. As soon as the layer was solidified under UV radiation the next layer is deposited.

To demonstrate the performance of the optimized metasurface, we printed an array of hemispheres with diameter of 300 and $440 \mu \mathrm{m}$ arranged in the square lattice with a period of $600 \mu \mathrm{m}$ on the silica substrate, $0.53 \mathrm{~mm}$ thick.

\subsection{Graphene Synthesis and Transfer Fabrication of the Metasurface}

We employ CVD graphene synthesized onto $25 \mu \mathrm{m}$ thick copper foil (Alfa Aesar, Ward Hill, MA, USA, 99.9\%) in the Carbolite Gero oven. Prior to the start of the synthesis process, the system was pumped out for $1 \mathrm{~h}$ to pressure of $0.1 \mathrm{mbar}$, then pumped at presence of $\mathrm{N}_{2} / \mathrm{H}_{2}(60 \mathrm{sccm})$ mixture for $1 \mathrm{~h}$, and after that was heated up to the working temperature of $1050^{\circ} \mathrm{C}$ at the rate of $20^{\circ} \mathrm{C} / \mathrm{min}$. The parameters of the graphene synthesis process are the following: temperature at dynamic regime is $1050{ }^{\circ} \mathrm{C}$, pressure is $4-4.2 \mathrm{mBar}$, the $\mathrm{CH}_{4}$ and $\mathrm{H}_{2}$ gas flow is $60 \mathrm{sccm}$, synthesis time is $120 \mathrm{~min}$.

The graphene sheet synthesized on copper foil was covered with a $200 \mathrm{~nm}$ think PMMA layer, which is needed for transfer purposes. The covalent bonding of polymer to carbon atoms in the graphene sheet may result in extra doping of graphene, i.e., to 
changing its surface conductivity [32]. The typical conductivity of the CVD graphene, which was synthesized on copper foil and then transferred to the dielectric substrate by using PMMA film, is about $1.1 \mathrm{mS}$ [33].

The copper foil was then removed by wet etching in the 30:1:2.5 $\mathrm{H}_{2} \mathrm{O}: \mathrm{H}_{2} \mathrm{O}_{2}: \mathrm{HCl}$ solution during $2 \mathrm{~h}$. After rinsing in water for one hour the PMMA/graphene bilayer is transferred to surface of the silica substrate with imprinted polymer hemispheres, which were pre-covered with $30 \mathrm{~nm}$ thin layer of nickel via either thermal evaporation or magnetron sputtering (see Figure 3a for schematic representation of the metasurface fabrication process and Figure $3 \mathrm{~b}$ presenting the photo of fabricated structures).

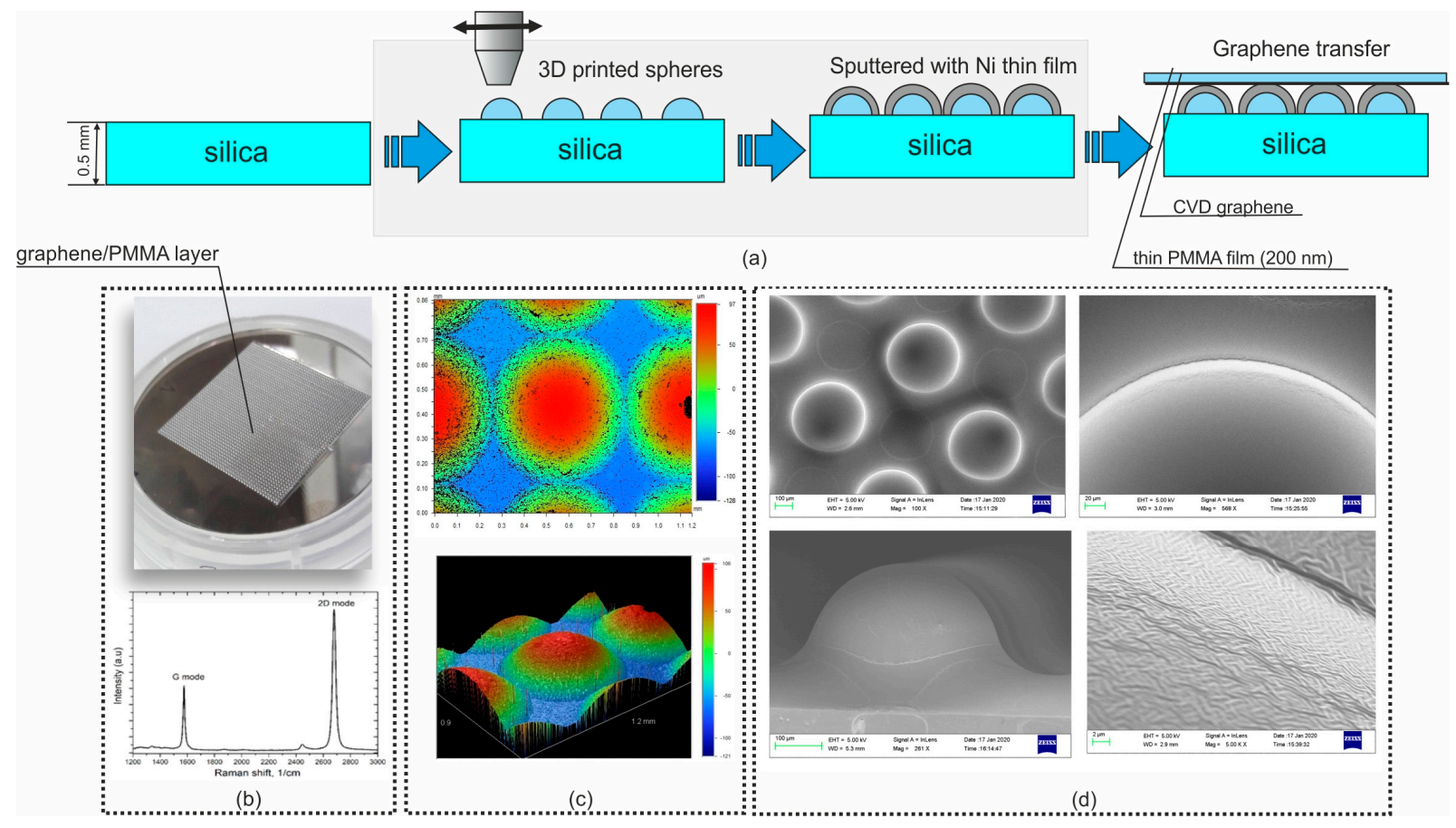

Figure 3. (a) Schematic presentation of the graphene enhanced structure fabrication using high-resolution 3D printing. (b) Photographic image of the fabricated structure and Raman spectrum of CVD graphene transferred on $\mathrm{Si} / \mathrm{SiO} \mathrm{S}_{2}(300 \mathrm{~nm})$ substrate for characterization. (c) Surface profile of the fabricated structure. (d) SEM images of 3D printed polymer hemispheres covered with $\mathrm{Ni}$ at different magnifications.

\subsection{Structural Characterization}

The surface topology and roughness of the polymer/Ni 3D printed structure were monitored by the optical profilometer Dektak 6M (Veeco Instruments, Plainview, NY, USA) and by Scanning electron microscopy (SEM-LEO 1550 Gemini, Zeiss, Jena, Germany), as presented in Figure 3c,d, respectively.

The quality of CVD graphene was controlled by Raman spectroscopy, HORIBA XploRA PLUS System, France, at $15 \mathrm{~s}$ exposure time at $\times 100$ magnification, using power below $0.8 \mathrm{~mW}$ of $532 \mathrm{~nm}$ laser for excitation, see Figure $3 \mathrm{~b}$. The sharp $\mathrm{G}$ peak in the vicinity of $1576 \mathrm{~cm}^{-1}$ (FWHM $21 \mathrm{~cm}^{-1}$ ) and Lorentz shape of 2D peak (peak at $2669 \mathrm{~cm}^{-1}$ ) with FWHM $\sim 30 \mathrm{~cm}^{-1}$ indicate (or are inherent in) the single-layer graphene. Furthermore, the absence of D peak in the vicinity of $1360 \mathrm{~cm}^{-1}$ emphasizes a high crystallinity of our CVD graphene.

\section{Experimental Results}

Free space transmittance $(T)$ and reflectance $(R)$ in THz range was measured using $\mathrm{THz}$ time-domain spectrometer (T-Spec, EKSPLA, Vilnius, Lithuania), see the measurement details in [33]. The samples were places by the face side (Ni or graphene/Ni first) towards the $3 \mathrm{~mm}$ THz beam treated as a plane wave as shown in Figure 4a. 


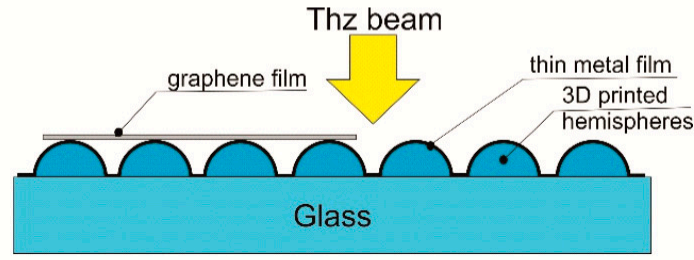

(a)

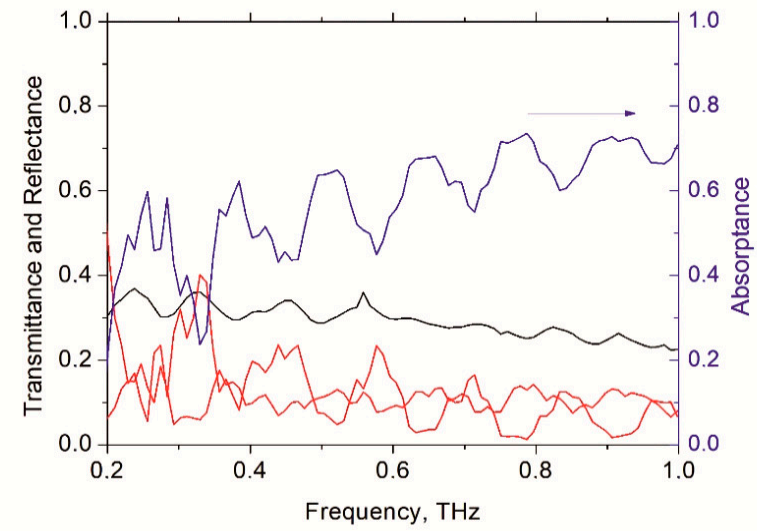

(c)

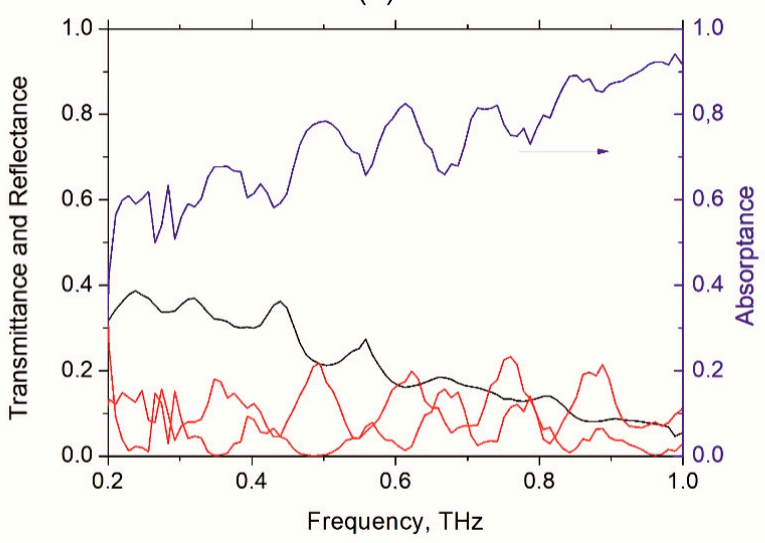

(e)

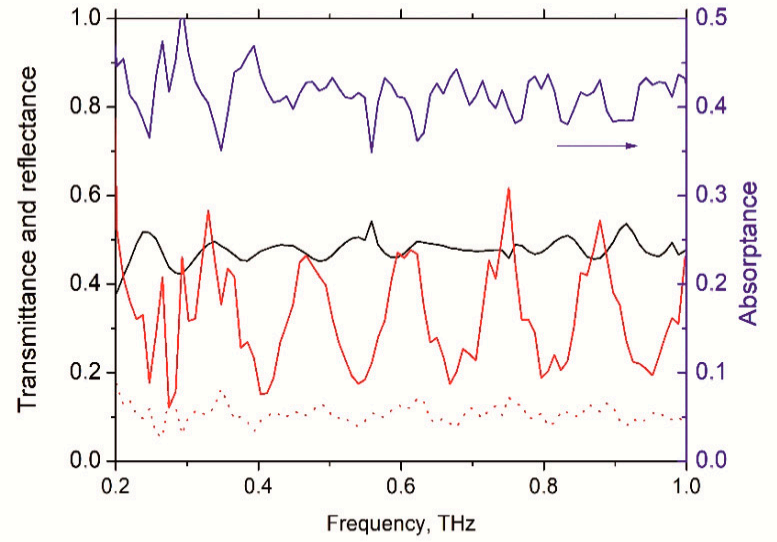

(b)

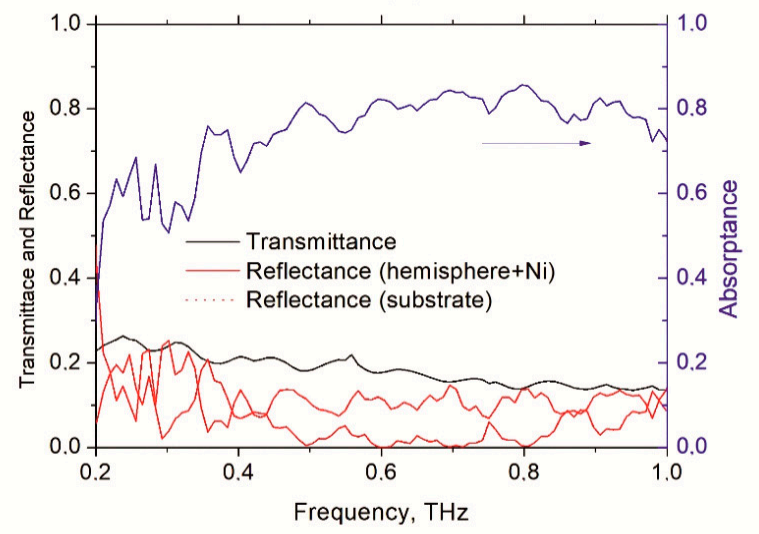

(d)

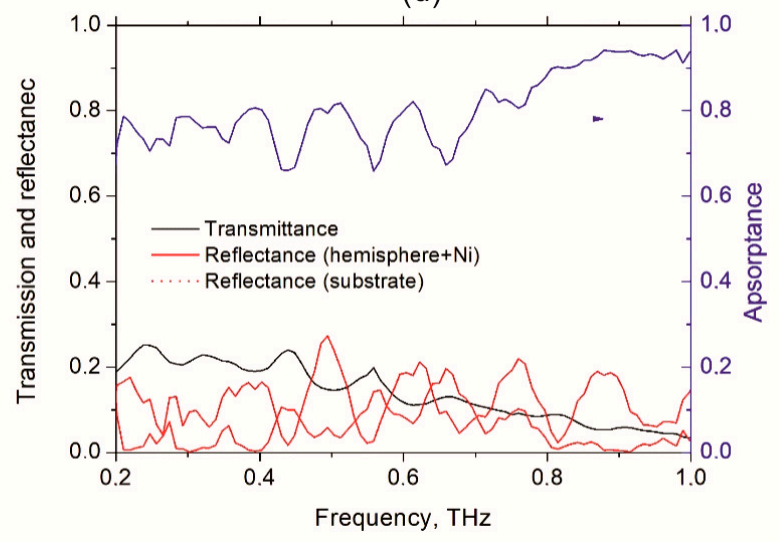

(f)

Figure 4. (a) Schematic presentation of the experiment. (b) Transmittance $(T)$, reflectance $(R)$ and absorptance $(A)$ spectra of the $30 \mathrm{~nm}$ thick $\mathrm{Ni}$ film deposited on plane $\mathrm{SiO}_{2}$ substrate; (c,e) show $T, R$ and $A$ spectra of $\mathrm{SiO}_{2} \backslash$ polymer hemispheres $\backslash$ nickel structures composed of hemispheres having diameters $300 \mu \mathrm{m}$ and $440 \mu \mathrm{m}$, respectively; (d) and (f) show the same spectra for $\mathrm{SiO}_{2} \backslash$ polymer hemispheres $\backslash$ nickel $\backslash$ graphene structures.

Figure 4c,e show the measured spectra of the transmittance, reflectance and reconstructed absorptance $(A)$ of the fabricated $\mathrm{Ni}$ / polymer hemispheres/substrate metasurfaces, whereas Figure $4 \mathrm{~d}$,f present the electromagnetic response of graphene/Ni/polymer hemispheres/substrate structures. The spectra for Ni layer, $30 \mathrm{~nm}$ thick, deposited on silica substrate are presented for comparison (Figure $4 b$ ). 


\section{Discussion and Conclusions}

One may see from Figure $4 \mathrm{~b}$ that the $30 \mathrm{~nm}$ thick Ni layer deposited on silica substrate absorbs not more than $40-45 \%$ energy of the incident $\mathrm{THz}$ radiation. It is worth noting that this result well corresponds to the fact that maximum absorptance of the free-standing metal film does not exceed 50\% [34]. However, our experimental and theoretical results show that structuring of the surface can considerably increase absorptance. Specifically, spectra in Figures 2a and 4c,e show that metasurface composed of polymer hemispheres having optimized diameters that arranged in the square lattice with the period of $600 \mu \mathrm{m}$ and covered $30 \mathrm{~nm}$ thick Ni layer can absorb much more than $50 \%$ of the incident radiation.

Figure $4 \mathrm{c}, \mathrm{e}$ and Figure $4 \mathrm{~d}, \mathrm{f}$ show $T, R$ and $A$ of the metasurfaces without and with graphene, respectively. It is important to note that metasurfaces without graphene demonstrate pronounced frequency dependence of the $T, R$, and $A$ having absorptance approaching $80-90 \%$ only at frequencies close to $1 \mathrm{THz}$, as it is expected from the modeling results (see Figure 1). The placing graphene sheet onto the $\mathrm{SiO}_{2} \backslash$ polymer hemispheres $\backslash$ Ni metasurface allows us to increase absorptance in the whole frequency range (Figures $2 \mathrm{~b}$ and $4 \mathrm{~d}, \mathrm{f}$ for modeling and experimental data correspondently). Moreover, the metasurface having hemispheres' diameter of 440 microns demonstrates absorptance of above $80 \%$ at $0.2-1 \mathrm{THz}$.

One can conclude that replacing flat substrate with 3D structured one can increase the absorptance level from $50 \%$ to $80-90 \%$. The presence of an additional graphene layer in the system makes it possible to significantly change the dispersion of the electromagnetic response and increase the overall absorption level, particularly at low frequencies.

Author Contributions: A.P. and N.V., THz measurements and data analysis, electromagnetic modeling; M.K. and M.B., samples fabrication, Raman characterization; M.P., 3D printing; Y.S., data acquisition, analysis, paper revision and editing; G.V. and S.M., data analysis and paper revision; P.K., task statement, conceptualization, paper writing. All authors have read and agreed to the published version of the manuscript.

Funding: This work is supported by the Academy of Finland via Flagship Programme Photonics Research and Innovation (PREIN) (decision no. 320166) and also via grants nos. 343393 and 323724, H2020 MSCA RISE projects Chartist, Grant agreement ID: 101007896, and DISETCOM, Grant agreement ID: 823728, NATO SPS Project *G5777 *2DSENSE”. P.K. is supported by Horizon 2020 IF TURANDOT project 836816.

Institutional Review Board Statement: Not applicable.

Informed Consent Statement: Not applicable.

Data Availability Statement: The raw data are available upon request from authors.

Acknowledgments: We are thankful to Olga Svirko (UEF) for laboratory assistance.

Conflicts of Interest: The authors declare no conflict of interest.

\section{References}

1. Kuzhir, P.; Paddubskaya, A.; Volynets, N.; Batrakov, K.; Kaplas, T.; Lamberti, P.; Kotsilkova, R.; Lambin, P. The main principles of passive devices based on graphene and carbon films in microwave-THz frequency range. J. Nanophotonics 2017, 11, 032504. [CrossRef]

2. Efetov, D.K.; Shiue, R.-J.; Gao, Y.; Skinner, B.; Walsh, E.D.; Choi, H.; Zheng, J.; Tan, C.; Crosso, G.; Peng, C.; et al. Fast thermal relaxation in cavity-coupled graphene bolometers with a Johnson noise read-out. Nat. Nanotechnol. 2018, 13, 787-801. [CrossRef]

3. Blaikie, A.; Miller, D.; Alemán, B.J. A fast and sensitive room-temperature graphene nanomechanical bolometer. Nat. Commun. 2019, 10, 4726. [CrossRef] [PubMed]

4. Watts, C.M.; Liu, X.; Padilla, W.J. Metamaterial electromagnetic wave absorbers. Adv. Mater. 2012, 24, 98-120. [CrossRef] [PubMed]

5. Dhillon, S.S.; Vitiello, M.S.; Linfield, E.H.; Davies, A.G.; Hoffmann, M.C.; Booske, J.; Paoloni, C.; Gensch, M.; Weightman, P.; Williams, G.P.; et al. The 2017 terahertz science and technology roadmap. J. Phys. D Appl. Phys. 2017, 50, 043001. [CrossRef]

6. Meisak, D.; Gurnevich, E.; Plyushch, A.; Bychanok, D.; Georgiev, V.; Kotsilkova, R.; Kuzhir, P. Robust design of compact microwave absorbers and waveguide matched loads based on DC-conductive 3D-printable filament. J. Appl. Phys. D 2020, 53, 305301. [CrossRef] 
7. Chung, B.-K.; Chuah, H.-T. Modeling of RF absorber for application in the design of anechoic chamber. Prog. Electromagn. Res. 2003, 43, 273-285. [CrossRef]

8. Ren, J.; Yin, J.Y. 3D-Printed low-cost dielectric-resonator-based ultra-broadband microwave absorber using carbon-loaded acrylonitrie butadiene styrene polymer. Materials 2018, 11, 1249. [CrossRef] [PubMed]

9. Kim, D.S.; Kim, D.J.; Kim, D.H.; Hwang, S.; Jang, J.H. Simple fabrication of an antireflective hemispherical surface structure using a self-assembly method for the terahertz frequency range. Opt. Lett. 2012, 37, 2742-2744. [CrossRef]

10. Batrakov, K.; Kuzhir, P.; Maksimenko, S.; Paddubskaya, A.; Voronovich, S.; Lambin, P.; Kaplas, T.; Svirko, Y. Flexible transparent graphene/polymer multilayers for efficient electromagnetic field absorption. Sci. Rep. 2014, 4, 7191. [CrossRef] [PubMed]

11. Wu, Z.S.; Ren, W.; Gao, L.; Zhao, J.; Chen, Z.; Liu, B.; Tang, D.; Yu, B.; Jiang, C.; Cheng, H.M. Synthesis of Graphene Sheets with High Electrical Conductivity and Good Thermal Stability by Hydrogen Arc Discharge Exfoliation. ACS Nano 2009, 3, $411-417$. [CrossRef]

12. Gosling, J.H.; Makarovsky, O.; Wang, F.; Cottam, N.D.; Greenaway, M.T.; Patanè, A.; Wildman, R.D.; Tuck, C.J.; Turyanska, L.; Fromhold, T.M. Universal mobility characteristics of graphene originating from charge scattering by ionised impurities. Commun. Phys. 2021, 4, 1-8. [CrossRef]

13. Novoselov, K.S.; Geim, A.K.; Morozov, S.V.; Jiang, D.; Katsnelson, M.I.; Grigorieva, I.V.; Dubonos, S.V.; Firsov, A.A. Twodimensional gas of massless Dirac fermions in graphene. Nature 2005, 438, 197-200. [CrossRef] [PubMed]

14. Docherty, C.J.; Lin, C.T.; Joyce, H.J.; Nicholas, R.J.; Herz, L.M.; Li, L.J.; Johnston, M.B. Extreme sensitivity of graphene photoconductivity to environmental gases. Nat. Commun. 2012, 3, 1228. [CrossRef]

15. Tasolamprou, A.C.; Koulouklidis, A.D.; Daskalaki, C.; Mavidis, C.P.; Kenanakis, G.; Deligeorgis, G.; Viskadourakis, Z.; Kuzhir, P.; Tzortzakis, S.; Kafesaki, M.; et al. Experimental demonstration of ultrafast THz modulation in a graphene-dased thin film absorber through negative photoinduced conductivity. ACS Photonics 2019, 6, 720-727. [CrossRef] [PubMed]

16. Arik, K.; Abdollahramezani, S.; Khavasi, A. Polarization insensitive and broadband terahertz absorber using graphene disks. Plasmonics 2017, 12, 393-398. [CrossRef]

17. Li, X.; Cai, W.; An, J.; Kim, S.; Nah, J.; Yang, D.; Piner, R.; Velamakanni, A.; Jung, I.; Tutuc, E.; et al. Large-area synthesis of high-quality and uniform graphene films on copper foils. Science 2009, 324, 1312-1314. [CrossRef]

18. Lin, W.-H.; Chen, T.-H.; Chang, J.-K.; Taur, J.-I.; Lo, Y.-Y.; Lee, W.-L.; Chang, C.-S.; Su, W.-B.; Wu, C.-I. A direct and polymer-free method for transferring graphene grown by chemical vapor deposition to any substrate. ACS Nano 2014, 8, 1784-1791. [CrossRef]

19. Choi, J.; Kim, H.J.; Wang, M.C.; Leem, J.; King, W.P.; Nam, S. Three-dimensional integration of graphene via swelling, shrinking and adaptation. Nano Lett. 2015, 15, 4525-4531. [CrossRef]

20. Lanza, M.; Bayerl, A.; Gao, T.; Porti, M.; Nafria, M.; Jing, G.Y.; Zhang, Y.F.; Liu, Z.F.; Duan, H.L. Graphene-coated atomic force microscope tips for reliable nanoscale electrical characterization. Adv. Mater. 2012, 25, 1440-1444. [CrossRef] [PubMed]

21. Román-Manso, B.; Figueiredo, F.M.; Achiaga, B.; Barea, R.; Pérez-Coll, D.; Morelos Gómez, A.; Terrones, M.; Osendi, M.I.; Belmonte, M.; Miranzo, P. Electrically functional 3D architectured graphene/SiC composites. Carbon 2016, 100, 318-328. [CrossRef]

22. Kaplas, T.; Sharma, D.; Svirko, Y. Few-layer graphene synthesis on a dielectric substrate. Carbon 2012, 50, 1503-1509. [CrossRef]

23. Wang, H.; Hsu, A.L.; Palacios, T. Graphene Electronics for RF Applications. IEEE Microw. Mag. 2012, 13, 114-125. [CrossRef]

24. Chen, H.; Lu, W.B.; Liu, Z.G.; Geng, M.Y. Microwave programmable graphene metasurface. ACS Photonics 2020, 7, 1425-1435. [CrossRef]

25. Zhang, Y.; Li, T.; Chen, Q.; Zhang, H.; O’Hara, J.F.; Abele, E.; Taylor, A.J.; Chen, H.T.; Azad, A.K. Independently tunable dual-band perfect absorber based on graphene at mid-infrared frequencies. Sci. Rep. 2016, 5, 18463. [CrossRef]

26. Paddubskaya, A.; Demidenko, M.; Batrakov, K.; Valušis, G.; Kaplas, T.; Svirko, Y.; Kuzhir, P. Tunable perfect THz absorber based on the stretchable ultrathin carbon-polymer bilayer. Materials 2019, 12, 143. [CrossRef]

27. Andreev, V.G.; Vdovin, V.A.; Voronov, P.S. An experimental study of millimeter wave absorption in thin metal films. Tech. Phys. Lett. 2003, 29, 953-955. [CrossRef]

28. Buron, J.D.; Pizzocchero, F.; Jessen, B.S.; Booth, T.J.; Nielsen, P.F.; Hansen, O.; Hilke, M.; Whiteway, E.; Jepsen, P.U.; Boggild, P.; et al. Electrically continuous graphene from single crystal copper verified by terahertz conductance spectroscopy and micro four-point probe. Nano Lett. 2014, 14, 6348. [CrossRef]

29. Assefa, B.G.; Saastamoinen, T.; Biskop, J.; Kuittinen, M.; Turunen, J.; Saarinen, J. 3D printed plano-freeform optics for non-coherent discontinuous beam shaping. Opt. Rev. 2018, 25, 456-462. [CrossRef]

30. Assefa, B.G.; Saastamoinen, T.; Pekkarinen, M.; Biskop, J.; Nissinen, V.; Kuittinen, M.; Turunen, J.; Saarinen, J. Realizing freeform optics using 3D-printer for industrial based tailored irradiance distribution. OSA Contin. 2019, 2, 690-702. [CrossRef]

31. Assefa, B.G.; Pekkarinen, M.; Partanen, H.; Biskop, J.; Turunen, J.; Saarinen, J. Imaging-quality 3D-printed centimeter-scale lens. Opt. Express 2019, 27, 12630. [CrossRef] [PubMed]

32. Reina, A.; Jia, X.; Ho, J.; Nezich, D.; Son, H.; Bulovic, V.; Dresselhaus, M.S.; Kong, J. Large area, few-layer graphene films on arbitrary substrates by chemical vapor deposition. Nano Lett. 2009, 9, 30-35. [CrossRef] [PubMed]

33. Batrakov, K.; Kuzhir, P.; Maksimenko, S.; Volynets, N.; Voronovich, S.; Paddubskaya, A.; Valusis, G.; Kaplas, T.; Svirko, Y.; Lambin, P. Enhanced microwave-to-terahertz absorption in graphene. Appl. Phys. Lett. 2016, 108, 123101. [CrossRef]

34. Jackson, J.D. Classical Electrodynamics, 3rd ed.; Publisher John Wiley\& Sons: Hoboken, NJ, USA, 1998. 